\title{
On Variational Properties of Quadratic Curvature Functionals
}

\author{
SHENG Weimin and WANG Lisheng
}

\begin{abstract}
In this paper, we investigate a class of quadratic Riemannian curvature functionals on closed smooth manifold $M$ of dimension $n \geq 3$ on the space of Riemannian metrics on $M$ with unit volume. We study the stability of these functionals at the metric with constant sectional curvature as its critical point.
\end{abstract}

Keywords: Quadratic curvature functional, Variational, Transverse-traceless, Conformal variation, Stability

$\operatorname{MSC}(2000): 58 \mathrm{E} 11,53 \mathrm{C} 24$

\section{Introduction}

Let $M$ be an $n$-dimensional compact and smooth manifold, and $\mathscr{M}_{1}$ the space of smooth Riemannian metrics on $M$ with unit volume, i.e. $\mathscr{M}_{1}=\{g \in \mathscr{M}: \operatorname{vol}(g)=1\}$, where $\mathscr{M}$ is the space of smooth Riemannian metrics on $M$. A functional $F: \mathscr{M} \rightarrow R$ is called Riemannian if it is invariant under the action of the diffeomorphism group.

Recall the decomposition of Riemannian curvature tensor $\mathrm{Rm}$

$$
R m=W+\frac{1}{n-2} R i c \odot g-\frac{1}{(n-1)(n-2)} R g \odot g,
$$

where $W$, Ric and $R$ denote the Weyl curvature tensor, the Ricci tensor and the scalar curvature, respectively, and $\odot$ the Kulkarni-Nomizu product. From (1.1), we have,

$$
|R m|^{2}=|W|^{2}+\frac{4}{n-2}|R i c|^{2}-\frac{2}{(n-1)(n-2)} R^{2} .
$$

The basic quadratic curvature functionals are

$$
\mathcal{W}=\int_{M}|W|^{2} d V_{g}, \quad \rho=\int_{M}|R i c|^{2} d V_{g}, \quad \mathcal{S}=\int_{M} R^{2} d V_{g} .
$$

From the decomposition formula (1.2), one has

$$
\mathcal{R}=\int_{M}|R m|^{2} d V_{g}=\int_{M}|W|^{2} d V_{g}+\frac{4}{n-2} \int_{M}|R i c|^{2} d V_{g}-\frac{2}{(n-1)(n-2)} \int_{M} R^{2} d V_{g} .
$$

\footnotetext{
${ }^{0}$ The authors were supported by NSFC 11571304.
} 
We point out that in dimension three, Weyl tensor vanishes, and in dimension four, the famous Chern-Gauss-Bonnet formula implies that $\mathcal{W}$ can be expressed as a linear combination of $\rho$ and $\mathcal{S}$ with the addition of a topological term. There are many results on these quadratic functionals. See [3, 4, 2, 12, for example. In [7, Gursky and Viaclovsky focus attention on a class of general quadratic curvature functionals

$$
\widetilde{\mathcal{F}}_{\tau}=(V o l)^{\frac{4-n}{n}}\left(\int_{M}|R i c|^{2} d V_{g}+\tau \int_{M}|R|^{2} d V_{g}\right)
$$

They investigate rigidity and stability properties of critical points of $\widetilde{\mathcal{F}}_{\tau}$ on the space of Riemannian metric space $\mathscr{M}$, and obtain a series of beautiful results.

In this paper, we study a class of more general quadratic curvature functionals:

$$
\mathcal{F}_{s, \tau}=\int_{M}|R m|^{2} d V_{g}+s \int_{M}|R i c|^{2} d V_{g}+\tau \int_{M} R^{2} d V_{g}
$$

on Riemannian metrics space $\mathscr{M}_{1}$, where $s, \tau$ are some constants.

Actually, $\mathcal{F}_{s, \tau}$ have been widely studied. We first introduce the following definition.

Definition 1.1 Let $M$ be a compact $n$-dimension manifold, a critical metric $g$ for $\mathcal{F}_{s, \tau}$ is called a local minimizer if for all metrics $\bar{g}$ in a $C^{2, \alpha}$-neighborhood of $g$, satisfying:

$$
\mathcal{F}_{s, \tau}[\bar{g}] \geq \mathcal{F}_{s, \tau}[g]
$$

We can also define the local maximizer for $\mathcal{F}_{s, \tau}$ by the same way.

In [11, Y. Muto studied Riemannian functional $I[g]=\int_{M}|R m|^{2} d V_{g}$ on $\mathscr{M}_{1}$, and proved when $M$ is a $C^{\infty}$ manifold diffeomorphic to $S^{n}$, the mapping $I: \mathscr{M}_{1} / \mathcal{D} \rightarrow R$ has a local minimum at the Riemannian metric $\bar{g}$ of positive constant sectional curvature, where $I: \mathscr{M}_{1} / \mathcal{D} \rightarrow R$ is a mapping deduced from $I: \mathscr{M}_{1} \rightarrow R$ and $\mathcal{D}$ is the diffeomorphism group of $M$ and $\mathscr{M}_{1} / \mathcal{D}$ is the space of orbits generated by $\mathcal{D}$ of Riemannian metrics. Recently, S. Maity [10 generalized the result for functional $\mathcal{R}_{p}(g)=\int_{M}|R m|^{p} d V_{g}$ on $\mathscr{M}_{1}$, she proved that for a compact Riemannian manifold $(M, g)$, if $g$ is either a spherical space form and $p \in[2, \infty)$, or a hyperbolic manifold and $p \in\left[\frac{n}{2}, \infty\right)$, then $g$ is strictly stable for $\mathcal{R}_{p}$. O. Kobayashi [8] investigated variational properties of the conformally invariant functional $v(g)=\frac{2}{n} \int_{M}|W|^{\frac{n}{2}}$, and derived that when $n=4, S^{2}(1) \times S^{2}(1)$ endowed with the standard Einstein metric $g$ is a strictly stable critical point of $v(g)$. In [6], X. Guo, H. Li and G. Wei developed the result to the case of dimension $n=6$.

In this paper, we concern the various properties for quadratic curvature functional $\mathcal{F}_{s, \tau}$. Before giving our results, we state some background knowledge.

Recall a canonical decomposition of tangent space of $\mathscr{M}$. Define the divergence operator $\delta_{g}$ : $S^{2}(M) \rightarrow T^{*} M$ by

$$
\left(\delta_{g} h\right)_{j}=g^{p q} h_{p j, q} .
$$

and $\delta_{g}^{*}: T^{*} M \rightarrow S^{2}(M)$ its $L^{2}$-adjoint operator

$$
\left(\delta_{g}^{*} \omega\right)_{i j}=-\frac{1}{2}\left(\omega_{i, j}+\omega_{j, i}\right),
$$


in local coordinates $\left\{e_{i}\right\}_{1 \leq i \leq n}$, where $h_{i j, k}=\nabla_{k} h_{i j}$ and $\omega_{i, j}=\nabla_{j} \omega_{i}, \nabla$ is the Riemannian covariant derivative of $(M, g)$. For a compact Riemannian manifold $M$, the tangent space of $\mathscr{M}$ at $g$, which is denoted by $T_{g} \mathscr{M}$ has the orthogonal decomposition (see [3] Lemma 4.57):

$$
T_{g} \mathscr{M}=S^{2}(M)=\left(I m \delta_{g}^{*}+C^{\infty}(M) \cdot g\right) \oplus\left(\delta_{g}^{-1}(0) \cap \operatorname{tr}_{\mathrm{g}}^{-1}(0)\right),
$$

where $\operatorname{Im} \delta_{g}^{*}$ is just the tangent space of the orbit of $g$ under the action of the group of diffeomorphisms of $M$. For $T_{g} \mathscr{M}_{1}=\left\{h \in S^{2}(M): \int_{M} \operatorname{tr}_{g}(h) d V_{g}=0\right\}$, we have:

$$
T_{g} \mathscr{M}_{1}=\left(\left(I m \delta_{g}^{*}+C^{\infty}(M) \cdot g\right) \cap T_{g} \mathscr{M}_{1}\right) \oplus\left(\delta_{g}^{-1}(0) \cap \operatorname{tr}_{\mathrm{g}}^{-1}(0)\right) .
$$

Definition 1.2 Let $h \in S^{2}(M)$, then $h$ is called transverse-traceless (TT for short) if $\delta_{g} h=0$ and $\operatorname{tr}_{\mathrm{g}} \mathrm{h}=0$.

On an Einstein manifold, by [9, we have the Lichnerowicz Laplacian

$$
\triangle_{L} h_{i j}=\triangle h_{i j}+2 R_{i k j l} h^{k l}-\frac{2}{n} R h_{i j}
$$

where $\triangle$ is the rough Laplacian, and $\triangle_{L}$ maps the space of transverse-traceless tensors to iteself (see [7] also). By use of [1.6, we can get following Propositions 1.1-1.2 easily.

Proposition 1.1 Let $(M, g)$ be a compact manifold with constant sectional curvature 1 . Then the least eigenvalue of the Lichnerowicz Laplacian on TT-tensors is $4 n$.

Proof. By (1.6), the Lichnerowicz Laplacian on TT tensor $h$ is

$$
\triangle_{L} h=\triangle h-2 n h .
$$

Then by use of the inequality

$$
0 \leq \int_{M}\left|h_{i j, k}+h_{j k, i}+h_{k i, j}\right|^{2} d V_{g}
$$

exchanging covariant derivatives and integrating by parts, we have that the least eigenvalue of the Lichnerowicz Laplacian on TT-tensors is $4 n$.

Proposition 1.2 Let $(M, g)$ be a compact manifold with constant sectional curvature -1 . Then the least eigenvalue of the Lichnerowicz Laplacian on TT-tensors is bounded below by $-n$.

Proof. Make use of the inequality

$$
\int_{M}\left|h_{i j, k}-h_{i k, j}\right|^{2} d V_{g} \geq 0
$$

we can get the result in the same way.

It can be easily to see (Corollary 2.2 below) that the metric $g$ with constant sectional curvature must be a critical point of $\mathcal{F}_{s, \tau}$. In this paper we study the stability of $\mathcal{F}_{s, \tau}$ at the critical point $g$ with constant sectional curvature. We have the following 
Theorem 1.1 Let $(M, g)$ be a $n$-dimensional compact manifold with constant sectional curvature $\lambda=1$, then restricted to transverse-traceless variations, the following hold:

(1) If $s>-4, \tau<\frac{6 n-12}{n(n-1)}$, the second variation of $\mathcal{F}_{s, \tau}$ on $(M, g)$ is non-negative. Therefore $\mathcal{F}_{s, \tau}$ gets its local minimum in TT directions;

(2) If $s<-4, \tau>\frac{6 n-12}{n(n-1)}$, the second variation of $\mathcal{F}_{s, \tau}$ on $(M, g)$ is non-positive. Therefore $\mathcal{F}_{s, \tau}$ gets its local maximum in TT directions.

Theorem 1.2 Let $(M, g)$ be a $n$-dimensional compact manifold with constant sectional curvature $\lambda=-1$, then restricted to transverse-traceless variations, the following hold:

(1) If $s>-4, \tau>\frac{6 n-12}{n(n-1)}$, the second variation of $\mathcal{F}_{s, \tau}$ on $(M, g)$ is non-negative. Therefore $\mathcal{F}_{s, \tau}$ gets its local minimum in TT directions;

(2) If $s<-4, \tau<\frac{6 n-12}{n(n-1)}$, the second variation of $\mathcal{F}_{s, \tau}$ on $(M, g)$ is non-positive. Therefore $\mathcal{F}_{s, \tau}$ gets its local maximum in TT directions.

Theorem 1.3 Let $(M, g)$ be a compact manifold with constant sectional curvature $\lambda=0$. Then the second variation of $\mathcal{F}_{s, \tau}$ at $g$ is non-negative as $s>-4$ and non-positive as $s<-4$ when the variation is restricted in $T T$ directions.

Theorem 1.4 Let $(M, g)$ be a compact manifold with constant sectional curvature $\lambda=0$. Then the second variation of $\mathcal{F}_{s, \tau}$ at $(M, g)$ is nonnegative as $s+4 \tau>\frac{4}{n}(\tau-1)$ and non-positive as $s+4 \tau<\frac{4}{n}(\tau-1)$ when the variation is restricted in the conformal directions.

We denote $\mathcal{M}_{1}([g])$ the space of unit volume metrics conformal to $g$.

Theorem 1.5 Let $(M, g)$ be an $n$-dimensional compact manifold with constant sectional curvature $\lambda=1$. Then the following hold:

(1) If $n=4, s+3 \tau>-1$, then $\mathcal{F}_{s, \tau}$ attains a local minimizer at $g$ in $\mathcal{M}_{1}([g])$. If $s+3 \tau<-1$, then $g$ is a local maximizer in $\mathcal{M}_{1}([g])$.

(2) If $n=3, \tau<1$ and $s>-\frac{8}{3} \tau-\frac{4}{3}$, or $\tau>1$ and $s>-\frac{12}{5} \tau-\frac{8}{5}$, then $\mathcal{F}_{s, \tau}$ attains a local minimizer at $g$ in $\mathcal{M}_{1}([g])$. If if $\tau<1$ and $s<-\frac{12}{5} \tau-\frac{8}{5}$, or $\tau>1$ and $s<-\frac{8}{3} \tau-\frac{4}{3}$, then $g$ is a local maximizer in $\mathcal{M}_{1}([g])$.

(3) When $n \geq 5$, then $\mathcal{F}_{s, \tau}$ attains a local minimizer at $g$ in $\mathcal{M}_{1}([g])$ if

$$
\left\{\begin{array} { l } 
{ \tau > \frac { 2 } { ( n - 1 ) ( n - 2 ) } } \\
{ s > - \frac { 4 ( n - 1 ) } { n } \tau - \frac { 4 } { n } }
\end{array} \quad \text { or } \quad \left\{\begin{array}{l}
\tau<\frac{2}{(n-1)(n-2)} \\
s>-\frac{2 n(n-1)}{3 n-4} \tau-\frac{8}{3 n-4} .
\end{array}\right.\right.
$$

and $g$ is a local maximizer in $\mathcal{M}_{1}([g])$ if

$$
\left\{\begin{array} { l } 
{ \tau > \frac { 2 } { ( n - 1 ) ( n - 2 ) } } \\
{ s < - \frac { 2 n ( n - 1 ) } { 3 n - 4 } \tau - \frac { 8 } { 3 n - 4 } }
\end{array} \quad \text { or } \quad \left\{\begin{array}{l}
\tau<\frac{2}{(n-1)(n-2)} \\
s<-\frac{4(n-1)}{n} \tau-\frac{4}{n} .
\end{array}\right.\right.
$$


Theorem 1.6 Let $(M, g)$ be an $n$-dimensional compact manifold with constant sectional curvature $\lambda=-1$, the following hold:

(1) If $n=4, s+3 \tau>-1$, Then $\mathcal{F}_{s, \tau}$ attains a local minimizer at $g$ in $\mathcal{M}_{1}([g])$. If $s+3 \tau<-1$, then $g$ is a local maximizer in $\mathcal{M}_{1}([g])$.

(2) If $n=3, \tau<1$ and $s>-\frac{8}{3} \tau-\frac{4}{3}$, or $\tau>1$ and $s>-\frac{12}{5} \tau-\frac{8}{5}$, then $\mathcal{F}_{s, \tau}$ attains a local minimizer at $g$ in $\mathcal{M}_{1}([g])$. If $\tau<1$ and $s<-\frac{12}{5} \tau-\frac{8}{5}$, or $\tau>1$ and $s<-\frac{8}{3} \tau-\frac{4}{3}$, then $g$ is a local maximizer in $\mathcal{M}_{1}([g])$.

(3) When $n \geq 5$, then $\mathcal{F}_{s, \tau}$ attains a local minimizer at $g$ in $\mathcal{M}_{1}([g])$ if

$$
\left\{\begin{array}{l}
\tau<\frac{2}{(n-1)(n-2)} \\
-\frac{4(n-1)}{n} \tau-\frac{4}{n}<s<-n \tau-\frac{2}{n-1} .
\end{array}\right.
$$

and $g$ is a local maximizer in $\mathcal{M}_{1}([g])$ if

$$
\left\{\begin{array} { l } 
{ \tau < \frac { 2 } { ( n - 1 ) ( n - 2 ) } } \\
{ s > - n \tau - \frac { 2 } { n - 1 } }
\end{array} \quad \text { or } \quad \left\{\begin{array}{l}
\tau>\frac{2}{(n-1)(n-2)} \\
s>-\frac{4(n-1)}{n} \tau-\frac{4}{n} .
\end{array}\right.\right.
$$

\section{The first variation and Euler-Lagrange equation}

Suppose $(M, g)$ be a $n$-dimensional Riemannian manifold. We choose a local orthonormal frame $\left\{e_{1}, e_{2}, \ldots e_{n}\right\}$, in accordance with the dual coframe $\left\{\omega^{1}, \omega^{2}, \ldots \omega^{n}\right\}$. Throughout this paper, we always adopt the moving frame notation with respect to a chosen local orthonormal frame, and also the Einstein summation convention. Let $g \in \mathscr{M}$ be an arbitrary fixed metric and $g=g_{i j} \omega^{i} \otimes \omega^{j}$, $g^{i j}=\left(g_{i j}\right)^{-1}$. Given a tensor, we raise or lower an index by contracting the tensor with the metric tensor $g$.

We denote $\nabla$ as the covariant derivative, and write $R_{i j, k}=\nabla_{k} R_{i j}, R_{i j, k l}=\nabla_{l} \nabla_{k} R_{i j}$, the Laplacian $\triangle R_{i j}=g^{k l} R_{i j, k l}$ and so on. For any $(0,2)$ tensor $S$, the Ricci identities can be expressed as

$$
S_{i j, k l}-S_{i j, l k}=S_{p j} R_{p i k l}+S_{i p} R_{p j k l} .
$$

Let $g(t) \in \mathscr{M}_{1}$ be a smooth variation of $g$ with $g(0)=g$ which can be represented locally as $g_{i j}\left(x^{1}, \ldots, x^{n} ; t\right)$. We define a tensor field $h \in S^{2}(M)$ with $h:=\frac{d}{d t} g(t)$. For convenience, we write $(\cdot)^{\prime}$ to stand for $\frac{d}{d t}$. Then we have the following formulae

$$
\left(g_{i j}\right)^{\prime}=h_{i j}, \quad\left(g^{i j}\right)^{\prime}=-h^{i j} .
$$

Proposition 2.1 Let $g(t) \in \mathscr{M}_{1}$ is a smooth variation (2.1), then

$$
\int_{M} \operatorname{tr}_{g(t)} h d V_{g}=0, \quad \int_{M}\left(g^{i j} \frac{d^{2}}{{d t^{2}}^{2}} g_{i j}-|h|^{2}+\frac{1}{2} H^{2}\right) d V_{g}=0,
$$

where $|h|^{2}=h^{i j} h_{i j}, H=\operatorname{tr}_{\mathrm{g}(\mathrm{t})} \mathrm{h}=\mathrm{g}^{\mathrm{ij}} \mathrm{h}_{\mathrm{ij}}$.

Proof. Recall $g(t) \in \mathscr{M}_{1}$, we have $\int_{M} d V_{g}=1$, then we have

$$
0=\frac{d}{d t}\left(\int_{M} d V_{g}\right)=\int_{M} \frac{d}{d t}\left(d V_{g}\right)=\int_{M} \frac{1}{2} g^{i j}\left(g_{i j}\right)^{\prime} d V_{g}=\frac{1}{2} \int_{M} \operatorname{tr}_{\mathrm{g}(\mathrm{t})} \mathrm{hdV} \mathrm{g} .
$$


Differentiating the above equality, we get

$$
\int_{M}\left(g^{i j} \frac{d^{2}}{d t^{2}} g_{i j}-|h|^{2}+\frac{1}{2}\left(\operatorname{tr}_{\mathrm{g}(\mathrm{t})} \mathrm{h}\right)^{2}\right) d V_{g}=0
$$

This proves (2.2).

From (2.1), we get the variation of the Christoffel symbols

$$
\frac{d}{d t} \Gamma_{i j}^{k}=\frac{1}{2} g^{k l}\left(h_{i l, j}+h_{j l, i}-h_{i j, l}\right) .
$$

By use of (2.3), we can get the following variational formulae of Riemannaian curvature tensor, Ricci curvature tensor and scalar curvature directly.

Proposition 2.2 The variations of Riemannaian curvature tensor, Ricci curvature tensor and scalar curvature are expressed as

$$
\begin{aligned}
\left(R_{i j k}^{l}\right)^{\prime} & =\frac{1}{2} g^{p l}\left(h_{i p, k j}+h_{k p, i j}-h_{i k, p j}-h_{i p, j k}-h_{j p, i k}+h_{i j, p k}\right), \\
\left(R_{l i j k}\right)^{\prime} & =h_{l q} R_{i j k}^{q}+\frac{1}{2}\left(h_{i l, k j}+h_{k l, i j}-h_{i k, l j}-h_{i l, j k}-h_{j l, i k}+h_{i j, l k}\right), \\
\left(R_{i k}\right)^{\prime} & =\frac{1}{2}\left(h_{i, k j}^{j}+h_{k, i j}^{j}-\triangle h_{i k}-H_{, i k}\right), \\
R^{\prime} & =-h^{i j} R_{i j}+h_{, i j}^{i j}-\triangle H .
\end{aligned}
$$

Now, we compute the first variation of the quadratic curvature functional $\mathcal{F}_{s, \tau}$ restricting on Riemannian metrics space $\mathscr{M}_{1}$ and derive its Euler-Lagrange equation. At first, we compute the first variations of $\mathcal{R}, \rho$, and $\mathcal{S}$, respectively.

By Proposition 2.2, we have

$$
\begin{aligned}
\frac{d}{d t}|R m|^{2}= & \frac{d}{d t}\left(g^{p l} g^{q i} g^{r j} g^{s k} R_{p q r s} R_{l i j k}\right) \\
= & -R_{p}^{i j k} R_{l i j k} h^{p l}-R_{q}^{l j k} R_{l i j k} h^{q i}-R_{r}^{l i k} R_{l i j k} h^{r j}-R^{l i j}{ }_{s} R_{l i j k} h^{s k}+2 R^{l i j k} \frac{d}{d t} R_{l i j k} \\
= & -R_{p}^{i j k} R_{l i j k} h^{p l}-R_{q}^{l j k} R_{l i j k} h^{q i}-R_{r}^{l i k} R_{l i j k} h^{r j}-R^{l i j}{ }_{s} R_{l i j k} h^{s k}+2 R^{l i j k} h_{l q} R^{q}{ }_{i j k} \\
& +R^{l i j k}\left(h_{i l, k j}+h_{k l, i j}-h_{i k, l j}-h_{i l, j k}-h_{j l, i k}+h_{i j, l k}\right) \\
= & -R_{p}^{i j k} R_{l i j k} h^{p l}-R_{q}^{l j k} R_{l i j k} h^{q i}-R_{r}^{l i k} R_{l i j k} h^{r j}-R^{l i j}{ }_{s} R_{l i j k} h^{s k} \\
& +2 R^{l i j k} h_{l q} R_{i j k}^{q}+4 R^{l i j k} h_{i j, l k}, \\
\frac{d}{d t}|R i c|^{2}= & \frac{d}{d t}\left(g^{p i} g^{q k} R_{p q} R_{i k}\right) \\
= & -2 h^{p i} R_{p}^{k} R_{i k}+2 R^{i k} \frac{d}{d t} R_{i k} \\
= & -2 h^{p i} R_{p}{ }^{k} R_{i k}+R^{i k}\left(h_{i, k j}^{j}+h_{k, i j}^{j}-\triangle h_{i k}-H_{, i k}\right), \\
\frac{d}{d t} R^{2}= & 2 R\left(-h^{i j} R_{i j}+h^{i j}{ }_{i j}-\triangle H\right) .
\end{aligned}
$$


Integrating by parts, we get

$$
\begin{aligned}
\frac{d}{d t} \mathcal{R}= & \int_{M}\left(\frac{d}{d t}|R m|^{2}+\frac{1}{2}|R m|^{2} H\right) d V_{g} \\
= & \int_{M}\left(-R_{p}^{i j k} R_{l i j k} h^{p l}-R_{q}^{l j k} R_{l i j k} h^{q i}-R_{r}^{l i k} R_{l i j k} h^{r j}-R^{l i j}{ }_{s} R_{l i j k} h^{s k}\right. \\
& \left.+2 R^{l i j k} h_{l q} R^{q}{ }_{i j k}+4 R^{l i j k} h_{i j, l k}+\frac{1}{2}|R m|^{2} H\right) d V_{g} \\
= & \int_{M}\left(-2 R_{i}{ }^{p l k} R_{j p l k}+2 R_{, i j}-4 \triangle R_{i j}-4 R^{p l} R_{i p j l}+4 R_{j p} R_{i}^{p}+\frac{1}{2}|R m|^{2} g_{i j}\right) h^{i j} d V_{g}, \\
\frac{d}{d t} \rho= & \int_{M}\left(\frac{d}{d t}|R i c|^{2}+\frac{1}{2}|R i c|^{2} H\right) d V_{g} \\
= & \int_{M}\left(-2 h^{p i} R_{p}{ }^{k} R_{i k}+R^{i k}\left(h_{i, k j}^{j}+h_{k, i j}^{j}-\triangle h_{i k}-H_{, i k}\right)+\frac{1}{2}|R i c|^{2} H\right) d V_{g} \\
= & \int_{M}\left(-\triangle R_{i j}-2 R^{p l} R_{i p j l}+R_{, i j}-\frac{1}{2}(\triangle R) g_{i j}+\frac{1}{2}|R i c|^{2} g_{i j}\right) h^{i j} d V_{g}, \\
\frac{d}{d t} \mathcal{S}= & \int_{M}\left(\frac{d}{d t}|R|^{2}+\frac{1}{2}|R|^{2} H\right) d V_{g} \\
= & \int_{M}\left(2 R\left(-h^{i j} R_{i j}+h_{, i j}^{i j}-\triangle H\right)+\frac{1}{2}|R|^{2} H\right) d V_{g} \\
= & \int_{M}\left(2 R, i j-2(\triangle R) g_{i j}-2 R R_{i j}+\frac{1}{2} R^{2} g_{i j}\right) h^{i j} d V_{g} .
\end{aligned}
$$

Lemma 2.1 ([3]) The gradients of the functionals $\mathcal{R}, \rho, \mathcal{S}$, and $\mathcal{F}_{s, \tau}$ are given by the following equations.

$$
\begin{aligned}
(\nabla \mathcal{R})_{i j}= & -2 R_{i}{ }^{p l k} R_{j p l k}+2 R_{, i j}-4 \triangle R_{i j}-4 R^{p l} R_{i p j l}+4 R_{j p} R_{i}^{p}+\frac{1}{2}|R m|^{2} g_{i j}, \\
(\nabla \rho)_{i j}= & -\triangle R_{i j}-2 R^{p l} R_{i p j l}+R_{, i j}-\frac{1}{2}(\triangle R) g_{i j}+\frac{1}{2}|R i c|^{2} g_{i j} \\
(\nabla \mathcal{S})_{i j}= & 2 R_{, i j}-2(\triangle R) g_{i j}-2 R R_{i j}+\frac{1}{2} R^{2} g_{i j} \\
\left(\nabla \mathcal{F}_{s, \tau}\right)_{i j}= & -2 R_{i}^{p l k} R_{j p l k}+2 R_{, i j}-4 \triangle R_{i j}-4 R^{p l} R_{i p j l}+4 R_{j p} R_{i}^{p}+\frac{1}{2}|R m|^{2} g_{i j} \\
& +s\left(-\triangle R_{i j}-2 R^{p l} R_{i p j l}+R_{, i j}-\frac{1}{2}(\triangle R) g_{i j}+\frac{1}{2}|R i c|^{2} g_{i j}\right) \\
& +\tau\left(2 R_{, i j}-2(\triangle R) g_{i j}-2 R R_{i j}+\frac{1}{2} R^{2} g_{i j}\right) .
\end{aligned}
$$

By the Lagrangian multiplier method, a Riemannian metric $g \in \mathscr{M}_{1}$ is critical for $\mathcal{F}_{s, \tau} \mid \mathscr{M}_{1}$ if and only if it satisfies the equation

$$
\left(\nabla \mathcal{F}_{s, \tau}\right)_{i j}=c g_{i j}
$$

for some constant $c$. The Euler-Lagrange equations for $\mathcal{F}_{s, \tau} \mid \mathscr{M}_{1}$ is obtained after a simple computation.

Theorem 2.1 Let $M$ be a compact n-dimensional Riemannian manifold. Then the Euler-Lagrange 
equations of $\mathcal{F}_{s, \tau} \mid \mathscr{M}_{1}$ are

$$
\begin{array}{r}
-(4+s) \triangle R_{i j}+(2+s+2 \tau) R_{, i j}+\frac{2-2 \tau}{n} \triangle R g_{i j}-2 R_{i}{ }^{p l k} R_{j p l k}-(4+2 s) R^{p l} R_{i p j l} \\
+4 R_{j p} R_{i}^{p}-2 \tau R R_{i j}+\frac{2}{n}\left(|R m|^{2} g_{i j}+s|R i c|^{2} g_{i j}+\tau R^{2} g_{i j}\right)=0 \\
(n-4)\left(|R m|^{2}+s|R i c|^{2}+\tau|R|^{2}\right)-(4+n s+4(n-1) \tau) \triangle R=2 n c .
\end{array}
$$

In fact, (2.8) comes from (2.6) by taking trace on both sides. Substituting (2.8) into (2.6) we can get (2.7) directly.

From Theorem 2.1, we know that any compact Riemannian manifold $(M, g)$ with constant sectional curvature is a critical metric for $\mathcal{F}_{s, \tau}$ on $\mathscr{M}_{1}$. However, Einstein metric can not always be a critical point of these functionals.

Corollary 2.1 ([3, [5]) Restricting on $\mathscr{M}_{1}$, an Einstein metric $g$ is a critical point of $\mathcal{F}_{s, \tau}$ if and only if the metric $g$ satisfies

$$
R_{i}^{p l k} R_{j p l k}=\frac{1}{n}|R m|^{2} g_{i j}
$$

Corollary 2.2 Any metric with constant sectional curvature is a critical point of $\mathcal{F}_{s, \tau}$ restricted on $\mathscr{M}_{1}\left(M^{n}\right)$.

\section{Second variations on constant sectional curva- ture manifolds}

In this section, we derive the second variations of $\mathcal{F}_{s, \tau} \mid \mathscr{M}_{1}$ at the metric with constant sectional curvature. Suppose $(M, g)$ has constant sectional curvature, then for some constant $\lambda$,

$$
R_{i j k l}=\lambda\left(g_{i k} g_{j l}-g_{i l} g_{j k}\right)
$$

From (3.1), we also have

$$
R_{i j}=(n-1) \lambda g_{i j}, \quad R=n(n-1) \lambda .
$$

Following (2.5), the first variation of $\mathcal{F}_{s, \tau}$ is

$$
\begin{aligned}
\frac{d}{d t} \mathcal{F}_{s, \tau}= & \int_{M}\left(-2 R_{i}{ }^{p l k} R_{j p l k}+2 R_{, i j}-4 \triangle R_{i j}-4 R^{p l} R_{i p j l}+4 R_{j p} R_{i}^{p}+\frac{1}{2}|R m|^{2} g_{i j}\right. \\
& +s\left(-\triangle R_{i j}-2 R^{p l} R_{i p j l}+R_{, i j}-\frac{1}{2}(\triangle R) g_{i j}+\frac{1}{2}|R i c|^{2} g_{i j}\right) \\
& \left.+\tau\left(2 R_{, i j}-2(\triangle R) g_{i j}-2 R R_{i j}+\frac{1}{2} R^{2} g_{i j}\right)\right) h^{i j} d V_{g} .
\end{aligned}
$$


For convenience, we write $G=\nabla \mathcal{F}_{s, \tau}$,

$$
\begin{aligned}
G_{i j}= & -2 R_{i}{ }^{p l k} R_{j p l k}+2 R_{, i j}-4 \triangle R_{i j}-4 R^{p l} R_{i p j l}+4 R_{j p} R_{i}^{p}+\frac{1}{2}|R m|^{2} g_{i j} \\
& +s\left(-\triangle R_{i j}-2 R^{p l} R_{i p j l}+R_{, i j}-\frac{1}{2}(\triangle R) g_{i j}+\frac{1}{2}|R i c|^{2} g_{i j}\right) \\
& +\tau\left(2 R_{, i j}-2(\triangle R) g_{i j}-2 R R_{i j}+\frac{1}{2} R^{2} g_{i j}\right) .
\end{aligned}
$$

Then, we rewrite the first variation of $\mathcal{F}_{s, \tau}(3.3)$ as

$$
\frac{d}{d t} \mathcal{F}_{s, \tau}=\int_{M} G_{i j} h^{i j} d V_{g}
$$

Differentiating equality (3.5) again, by use of Proposition 2.1, we have

$$
\begin{aligned}
\left.\frac{d^{2}}{d t^{2}}\right|_{t=0} \mathcal{F}_{s, \tau}=\left.\frac{d}{d t}\right|_{t=0} \int_{M} G_{i j} h^{i j} d V_{g} \\
\quad=\left.\int_{M} \frac{d}{d t}\right|_{t=0}\left(G_{i j}\right) h^{i j} d V_{g}+\int_{M}\left(-2 c|h|^{2}+\left.c g^{i j} \frac{d^{2}}{d t^{2}}\right|_{t=0}\left(g_{i j}\right)\right) d V_{g}+\int_{M} \frac{1}{2} c H^{2} d V_{g} \\
\quad=\left.\int_{M} \frac{d}{d t}\right|_{t=0}\left(G_{i j}\right) h^{i j} d V_{g}-\int_{M} c|h|^{2} d V_{g} .
\end{aligned}
$$

Nextly, we concern the second variations on TT directions at some critical metric with constant sectional curvature.

\subsection{Transverse-traceless variations}

According to (3.4) and (3.6), we computer $\left.\frac{d}{d t}\right|_{t=0}\left(G_{i j}\right)$ at the metric $g$ with constant sectional curvature.

Proposition 3.1 If $g$ has constant sectional curvature, satisfying (3.1) and (3.2), then

$$
\begin{aligned}
\left(R_{i j, k}\right)^{\prime} & =\left(R_{i j}^{\prime}\right)_{, k}-\lambda(n-1) h_{i j, k}, \\
\left(R_{i j, k l}\right)^{\prime} & =\left(R_{i j}^{\prime}\right)_{, k l}-\lambda(n-1) h_{i j, k l}, \\
\left(\triangle R_{i j}\right)^{\prime} & =\left(\triangle R_{i j}^{\prime}\right)-\lambda(n-1) \triangle h_{i j}, \\
(\triangle R)^{\prime} & =\triangle\left(t r R i c^{\prime}\right)-\lambda(n-1) \triangle H .
\end{aligned}
$$

From Proposition 3.1, we calculate the second variations on TT direction.

$$
\begin{aligned}
\int_{M}\left(R_{i}{ }^{p l k} R_{j p l k}\right)^{\prime} h^{i j} d V g= & \int_{M}\left(\left(R_{i}{ }^{p l k}\right)^{\prime} R_{j p l k}+R_{i}{ }^{p l k}\left(R_{j p l k}\right)^{\prime}\right) h^{i j} d V_{g} \\
= & \int_{M}\left(-h^{p m} R_{i m k l} R_{j p k l}-h^{k n} R_{i p k l} R_{j p n l}-h^{l s} R_{i p k s} R_{j p k l}\right. \\
& \left.+2 R_{j k p l}\left(R_{i k p l}\right)^{\prime}\right) h^{i j} d V_{g} \\
= & \int_{M}\left((4-2 n) \lambda^{2} h_{i j}+2 R_{j p k l}\left(h_{i q} R_{q p k l}\right.\right. \\
& \left.\left.+\frac{1}{2}\left(h_{p i, l k}+h_{l i, p k}-h_{p l, i k}-h_{p i, k l}-h_{k i, p l}+h_{p k, i l}\right)\right)\right) h^{i j} d V_{g} \\
= & \int_{M}\left(2(n+1) \lambda^{2}|h|^{2}-2 \lambda h \triangle h\right) d V_{g} .
\end{aligned}
$$


By the same way, we can get the following formulae. Here we omit the detailed calculation.

$$
\begin{aligned}
\int_{M}\left(\triangle R_{i j}\right)^{\prime} h^{i j} d V_{g} & =\int_{M}\left(-\frac{1}{2} h \triangle^{2} h+\lambda h \triangle h\right) d V_{g}, \\
\int_{M}\left(R_{, i j}\right)^{\prime} h^{i j} d V_{g} & =0 \\
\int_{M}\left(R_{i}^{l} R_{j l}\right)^{\prime} h^{i j} d V_{g} & =\int_{M}\left(\lambda^{2}\left(n^{2}-1\right)|h|^{2}-\lambda(n-1) h \triangle h\right) d V_{g}, \\
\int_{M}\left(R^{p l} R_{i p j l}\right)^{\prime} h^{i j} d V_{g} & =\int_{M}\left(\left(n^{2}-n-1\right) \lambda^{2}|h|^{2}-\frac{1}{2} \lambda(n-2) h \triangle h\right) d V_{g}, \\
\int_{M}\left(|R m|^{2} g_{i j}\right)^{\prime} h^{i j} d V_{g} & =\int_{M} 2 \lambda^{2} n(n-1)|h|^{2} d V_{g}, \\
\int_{M}\left(\triangle R g_{i j}\right)^{\prime} h^{i j} d V_{g} & =0, \\
\int_{M}\left(|R i c|^{2} g_{i j}\right)^{\prime} h^{i j} d V_{g} & =\int_{M} \lambda^{2} n(n-1)^{2}|h|^{2} d V_{g}, \\
\int_{M}\left(R R_{i j}\right)^{\prime} h^{i j} d V_{g} & =\int_{M}\left(\lambda^{2} n^{2}(n-1)|h|^{2}-\frac{1}{2} \lambda n(n-1) h \triangle h\right) d V_{g}, \\
\int_{M}\left(R^{2} g_{i j}\right)^{\prime} h^{i j} d V_{g} & =\int_{M} \lambda^{2} n^{2}(n-1)^{2}|h|^{2} d V_{g} .
\end{aligned}
$$

Following (3.6) and the above equations, we can get the second variations of $\mathcal{F}_{s, \tau}$ in TT direction.

Theorem 3.1 Let $(M, g)$ be a compact Riemannian manifold with constant curvature satisfying (3.1) and $h$ a TT tensor. Then the second variation of $\mathcal{F}_{s, \tau}$ is

$$
\begin{aligned}
\left.\frac{d^{2}}{d t^{2}}\right|_{t=0} \mathcal{F}_{s, \tau} & =\int_{M}\left\langle\left( 2\left(\triangle_{L}+2(n-1) \lambda\right)\left(\triangle_{L}+(n+2) \lambda\right) h\right.\right. \\
& \left.+\frac{1}{2}\left(\triangle_{L}+2(n-1) \lambda\right)\left(s \triangle_{L}+(n-1)(4 s+2 n \tau) \lambda h\right)\right\rangle d V_{g} \\
= & \int_{M}\left\langle\left(\left(\triangle_{L}+2(n-1) \lambda\right)\left(\frac{4+s}{2} \triangle_{L}+\lambda(2 n+4+(n-1)(2 s+n \tau) \lambda)\right) h, h\right\rangle d V_{g} .\right.
\end{aligned}
$$

When $\lambda=0$, (3.7) becomes

$$
\left.\frac{d^{2}}{d t^{2}}\right|_{t=0} \mathcal{F}_{s, \tau}=\int_{M} 2\left(1+\frac{s}{4}\right) h \triangle^{2} h d V_{g}
$$

By (3.8), we can get the following corollary easily.

Corollary 3.1 Let $(M, g)$ be a compact manifold with constant sectional curvature $\lambda=0$. Then the second variation of $\mathcal{F}_{s, \tau}$ at $g$ is non-negative as $s>-4$ and non-positive as $s<-4$ when the variation is restricted on TT directions.

Now, we focus on the case $\lambda \neq 0$ and finish the proof of Theorems 1.1 and 1.2. Firstly, if $\lambda>0$, we set $\lambda=1$. We then have

$$
\left.\frac{d^{2}}{d t^{2}}\right|_{t=0} \mathcal{F}_{s, \tau}=\int_{M}\left\langle\left(\left(\triangle_{L}+2(n-1)\right)\left(\frac{4+s}{2} \triangle_{L}+(2 n+4)+(n-1)(2 s+n \tau)\right)\right) h, h\right\rangle d V_{g} .
$$


Proof of Theorem 1.1. By Proposition 1.1 we know the least eigenvalue of the Lichnerowicz Laplacian on TT tensors is $4 n$. Let $-\triangle_{L} h_{i j}=\lambda_{L} h_{i j}$, and rewrite (3.9) as

$$
\left.\frac{d^{2}}{d t^{2}}\right|_{t=0} \mathcal{F}_{s, \tau}=\int_{M}\left(\left(\lambda_{L}-2(n-1)\right)\left(\frac{4+s}{2} \lambda_{L}-2 n-4-(n-1)(2 s+n \tau)\right)\right)|h|^{2} d V_{g} .
$$

The first term $\lambda_{L}-2(n-1)>0$. We consider the second term. If $s>-4, \tau<\frac{6 n-12}{n(n-1)}$,

$$
\begin{aligned}
\frac{4+s}{2} \lambda_{L}-(2 n+4)-(n-1)(2 s+n \tau) & >\frac{4+s}{2} 4 n-(2 n+4)-(n-1)(2 s+n \tau) \\
& >6 n-12-n(n-1) \tau \\
& >0,
\end{aligned}
$$

So, in this case we have $\left.\frac{d^{2}}{d t^{2}}\right|_{t=0} \mathcal{F}_{s, \tau} \geq 0$. If $s<-4, \tau>\frac{6 n-12}{n(n-1)}$,

$$
\begin{aligned}
\frac{4+s}{2} \lambda_{L}-(2 n+4)-(n-1)(2 s+n \tau) & <\frac{4+s}{2} 4 n-(2 n+4)-(n-1)(2 s+n \tau) \\
& <6 n-12-n(n-1) \tau \\
& <0 .
\end{aligned}
$$

Therefore, in this case we have $\left.\frac{d^{2}}{d t^{2}}\right|_{t=0} \mathcal{F}_{s, \tau} \leq 0$.

Proof of Theorem 1.2. If $\lambda<0$, we set $\lambda=-1$. We have

$$
\begin{aligned}
\left.\frac{d^{2}}{d t^{2}}\right|_{t=0} \mathcal{F}_{s, \tau} & =\int_{M}\left(\left(\triangle_{L}-2(n-1)\right)\left(\frac{4+s}{2} \triangle_{L}-((2 n+4)-(n-1)(2 s+n \tau))\right) h d V_{g}\right. \\
& =\int_{M}\left(\left(\lambda_{L}+2(n-1)\right)\left(\frac{4+s}{2} \lambda_{L}+((2 n+4)+(n-1)(2 s+n \tau))\right)|h|^{2} d V_{g} .\right.
\end{aligned}
$$

By Proposition 1.2, the first term $\lambda_{L}+2(n-1)>0$. We now consider the second term. If $s>-4$, and $\tau>\frac{6 n-12}{n(n-1)}$,

$$
\begin{aligned}
\frac{4+s}{2} \lambda_{L}+(2 n+4)+(n-1)(2 s+n \tau) & >-\frac{4+s}{2} n+2 n+4+(n-1)(2 s+n \tau) \\
& >-6 n+12+n(n-1) \tau \\
& >0
\end{aligned}
$$

In this case we have $\left.\frac{d^{2}}{d t^{2}}\right|_{t=0} \mathcal{F}_{s, \tau} \geq 0$. By the same way we know $\left.\frac{d^{2}}{d t^{2}}\right|_{t=0} \mathcal{F}_{s, \tau} \geq 0$ when $s<-4$, $\tau<\frac{6 n-12}{n(n-1)}$.

\subsection{Conformal variations}

Now, we consider the conformal variations of the functionals $\mathcal{F}_{s, \tau}$ at a Riemannian metric with constant sectional curvature satisfying (3.1). Let $\mathcal{M}_{1}[g]$ denote the space of unit volume metrics conformal to $g$. The tangent space of $\mathcal{M}_{1}[g]$ consists of functionals with mean value zero. 
Proposition 3.2 If $g$ has constant sectional curvature, satisfying (3.1) and (3.2), and let $h=f g$,

$$
\begin{aligned}
\left(R_{i j}\right)^{\prime} & =-\frac{1}{2}(n-2) f_{, i j}-\frac{1}{2} \triangle f g_{i j}, \\
\left(R_{i j, k l}\right)^{\prime} & =\left(R_{i j}^{\prime}\right)_{, k l}-\lambda(n-1) f_{, k l} g_{i j}, \\
\left(\triangle R_{i j}\right)^{\prime} & =\left(\triangle R_{i j}^{\prime}\right)-\lambda(n-1) \triangle f g_{i j}, \\
(\triangle R)^{\prime} & =-(n-1) \triangle^{2} f-\lambda n(n-1) \triangle f .
\end{aligned}
$$

Following proposition (3.2), we have,

$$
\begin{aligned}
\int_{M}\left(R_{i}{ }^{p l k} R_{j p l k}\right)^{\prime} f g^{i j} d V g= & \int_{M}\left(\left(R_{i}{ }^{p l k}\right)^{\prime} R_{j p l k}+R_{i}{ }^{p l k}\left(R_{j p l k}\right)^{\prime}\right) h^{i j} d V_{g} \\
= & \int_{M}\left(-f g^{p m} R_{i m k l} R_{j p k l}-f g^{k n} R_{i p k l} R_{j p n l}-f g^{l s} R_{i p k s} R_{j p k l}\right. \\
& \left.+2 R_{j k p l}\left(R_{i k p l}\right)^{\prime}\right) f g^{i j} d V_{g} \\
= & \int_{M}\left(-3|R m|^{2} f^{2}+2 f R^{i k j l} R_{i k j l}{ }_{i k}\right) d V_{g} \\
= & \int_{M}\left(-3|R m|^{2} f^{2}+2 \lambda f\left(g^{i j} g^{k l}-g^{i l} g^{k j}\right) R_{i k j l}^{\prime}\right) d V_{g} \\
= & \int_{M}\left(-3|R m|^{2} f^{2}+4 \lambda f g^{i j}\left(\left(g^{k l} R_{i k j l}\right)^{\prime}+f g^{k l} R_{i k j l}\right)\right) d V_{g} \\
= & \int_{M}\left(-2 \lambda^{2} n(n-1) f^{2}-4 \lambda(n-1) f \triangle f\right) d V_{g} .
\end{aligned}
$$

By the same way, we give the following formulas.

$$
\begin{aligned}
\int_{M}\left(\triangle R_{i j}\right)^{\prime} f g^{i j} d V_{g} & =\int_{M}\left(-(n-1) f \triangle^{2} f-\lambda n(n-1) f \triangle f\right) d V_{g}, \\
\int_{M}\left(R_{, i j}\right)^{\prime} f g^{i j} d V_{g} & =\int_{M}\left(-(n-1) f \triangle^{2} f-\lambda n(n-1) f \triangle f\right) d V_{g}, \\
\int_{M}\left(R_{i}^{l} R_{j l}\right)^{\prime} f g^{i j} d V_{g} & =\int_{M}\left(-\lambda^{2} n(n-1)^{2} f^{2}-2 \lambda(n-1)^{2} f \triangle f\right) d V_{g}, \\
\int_{M}\left(R^{p l} R_{i p j l}\right)^{\prime} f g^{i j} d V_{g} & =\int_{M}\left(-\lambda^{2} n(n-1)^{2} f^{2}-2 \lambda(n-1)^{2} f \triangle f\right) d V_{g}, \\
\int_{M}\left(|R m|^{2} g_{i j}\right)^{\prime} f g^{i j} d V_{g} & =\int_{M}\left(-2 \lambda^{2} n^{2}(n-1) f^{2}-4 \lambda n(n-1) f \triangle f\right) d V_{g}, \\
\int_{M}\left(\triangle R g_{i j}\right)^{\prime} f g^{i j} d V_{g} & =\int_{M}\left(-n(n-1) f \triangle^{2} f-\lambda n^{2}(n-1) f \triangle f\right) d V_{g}, \\
\int_{M}\left(|R i c|^{2} g_{i j}\right)^{\prime} f g^{i j} d V_{g} & =\int_{M}\left(-\lambda^{2} n^{2}(n-1)^{2} f^{2}-2 \lambda n(n-1)^{2} f \triangle f\right) d V_{g}, \\
\int_{M}\left(R R_{i j}\right)^{\prime} f g^{i j} d V_{g} & =\int_{M}\left(-\lambda^{2} n^{2}(n-1)^{2} f^{2}-2 \lambda n(n-1)^{2} f \triangle f\right) d V_{g}, \\
\int_{M}\left(R^{2} g_{i j}\right)^{\prime} f g^{i j} d V_{g} & =\int_{M}\left(-\lambda^{2} n^{3}(n-1)^{2} f^{2}-2 \lambda n^{2}(n-1)^{2} f \triangle f\right) d V_{g},
\end{aligned}
$$


Using the above equalities, we get the conformal variations for $\mathcal{F}_{s, \tau}$ at metrics with constant sectional curvature.

Theorem 3.2 Let $(M, g)$ is a compact Riemannian manifold with constant curvature satisfying (3.1) and $h=f g$ with $\int_{M} f d V_{g}=0$. Then the second variation of $\mathcal{F}_{s, \tau}$ is

$$
\begin{aligned}
\left.\frac{d^{2}}{d t^{2}}\right|_{t=0} \mathcal{F}_{s, \tau} & =\int_{M}\left(2(n-1) f \triangle^{2} f+8 \lambda(n-1) f \triangle f-2 \lambda^{2}\left(n^{2}-n\right)(n-4) f^{2}\right. \\
& +s\left(\frac{1}{2} n(n-1) f \triangle^{2} f-\frac{1}{2} \lambda(n-1)\left(n^{2}-10 n+8\right) f \triangle f-\lambda^{2} n(n-1)^{2}(n-4) f^{2}\right) \\
& \left.+\tau\left(2(n-1)^{2} f \triangle^{2} f-\lambda n(n-1)^{2}(n-6) f \triangle f-\lambda^{2} n^{2}(n-1)^{2}(n-4) f^{2}\right)\right) d V g
\end{aligned}
$$

When $\lambda=0,(3.12)$ becomes

$$
\left.\frac{d^{2}}{d t^{2}}\right|_{t=0} \mathcal{F}_{s, \tau}=\int_{M} \frac{1}{2}(n-1)(s n+4(n-1) \tau+4) f \triangle^{2} f d V g .
$$

We then have

Corollary 3.2 Let $(M, g)$ be a compact manifold with constant sectional curvature $\lambda=0$. Then the second variation of $\mathcal{F}_{s, \tau}$ on $(M, g)$ is nonnegative as $s+4 \tau>\frac{4}{n}(\tau-1)$ when the variation is restricted on the conformal direction.

Next we consider the case $\lambda \neq 0$ and finish the proof of Theorems 1.5 and 1.6. Firstly, if $\lambda>0$, we set $\lambda=1$. We then have

$$
\begin{aligned}
\left.\frac{d^{2}}{d t^{2}}\right|_{t=0} \mathcal{F}_{s, \tau}=\int_{M}\left\langle( n - 1 ) ( \Delta + n ) \left(\frac{n s-4 \tau+4 n \tau+4}{2} \triangle\right.\right. \\
\left.\left.\quad-(n-4)\left(n^{2} \tau+n s-n \tau-s+2\right)\right) f, f\right\rangle d V_{g} .
\end{aligned}
$$

Proposition 3.3 (【1]) If $\left(M^{n}, g\right)$ is a compact manifold satisfying

$$
R i c \geq(n-1) \cdot g,
$$

then the lowest non-trivial eigenvalue satisfies $\lambda_{1} \geq n$, and the equality holds if and any if $\left(M^{n}, g\right)$ is isometric to $\left(S^{n}, g_{s}\right)$.

Let $\mu$ be a non-zero eigenvalue of $\triangle$. We consider the following polynomial

$$
P_{1}(\mu)=(n-1)(\mu-n)\left(\frac{n s-4 \tau+4 n \tau+4}{2} \mu+(n-4)\left(n^{2} \tau+n s-n \tau-s+2\right)\right) .
$$

Since the variation is restricted to $\mathcal{M}_{1}[g]$, we should to prove that the second variation of the functional on functions with mean value zero is non-negative (or non-positive in the maximizing case).

Proof of Theorem 1.5. By Proposition (3.3), the second term $\mu-n \geq 0$. Then the sign of the second variations for $\mathcal{F}_{s, \tau}$ is determined by the third term. If $n=4$,

$$
P_{1}(\mu)=6(\mu-4)(s+3 \tau+1) \mu .
$$


Form (3.16), the first part of Theorem 1.5 can get easily.

If $n=3$,

$$
P_{1}(\mu)=2(\mu-3)\left(\frac{3 s+8 \tau+4}{2} \mu-(6 \tau+2 s+2)\right) .
$$

The functional will be minimizing if $\frac{3 s+8 \tau+4}{2}>0$ and

$$
\frac{3 s+8 \tau+4}{2} \mu-(6 \tau+2 s+2) \geq \frac{5}{2} s+6 \tau+4>0 .
$$

Then $P_{1}(\mu) \geq 0$ if the following hold:

$$
\left\{\begin{array} { l } 
{ \tau < 1 } \\
{ s > - \frac { 8 } { 3 } \tau - \frac { 4 } { 3 } }
\end{array} \quad \text { or } \quad \left\{\begin{array}{l}
\tau>1 \\
s>-\frac{12}{5} \tau-\frac{8}{5} .
\end{array}\right.\right.
$$

Similarly we can get $P_{1}(\mu) \leq 0$ if the following hold:

$$
\left\{\begin{array} { l } 
{ \tau < 1 } \\
{ s < - \frac { 1 2 } { 5 } \tau - \frac { 8 } { 5 } }
\end{array} \quad \text { or } \quad \left\{\begin{array}{l}
\tau>1 \\
s<-\frac{8}{3} \tau-\frac{4}{3} .
\end{array}\right.\right.
$$

When $n \geq 5$, the functional will be minimizing if $n s-4 \tau+4 n \tau+4>0$ and

$$
\frac{n s-4 \tau+4 n \tau+4}{2} n+(n-4)\left(n^{2} \tau+n s-n \tau-s+2\right)>0 .
$$

Then $P_{1}(\mu) \geq 0$ if the following hold

$$
\left\{\begin{array} { l } 
{ \tau > \frac { 2 } { ( n - 1 ) ( n - 2 ) } } \\
{ s > - \frac { 4 ( n - 1 ) } { n } \tau - \frac { 4 } { n } }
\end{array} \quad \text { or } \quad \left\{\begin{array}{l}
\tau<\frac{2}{(n-1)(n-2)} \\
s>-\frac{2 n(n-1)}{3 n-4} \tau-\frac{8}{3 n-4} .
\end{array}\right.\right.
$$

And we can get $P_{1}(\mu) \leq 0$ if the following hold

$$
\left\{\begin{array} { l } 
{ \tau > \frac { 2 } { ( n - 1 ) ( n - 2 ) } } \\
{ s < - \frac { 2 n ( n - 1 ) } { 3 n - 4 } \tau - \frac { 8 } { 3 n - 4 } }
\end{array} \quad \text { or } \quad \left\{\begin{array}{l}
\tau<\frac{2}{(n-1)(n-2)} \\
s<-\frac{4(n-1)}{n} \tau-\frac{4}{n} .
\end{array}\right.\right.
$$

Finally we consider the case $\lambda<0$. We set $\lambda=-1$.

$$
\begin{aligned}
\left.\frac{d^{2}}{d t^{2}}\right|_{t=0} \mathcal{F}_{s, \tau}=\int_{M}\langle(n-1)( & -n)\left(\frac{n s-4 \tau+4 n \tau+4}{2} \triangle\right. \\
& \left.\left.+(n-4)\left(n^{2} \tau-n \tau-s+2\right)\right) f, f\right\rangle d V_{g} .
\end{aligned}
$$

Let $\mu$ is a non-zero eigenvalue of $\triangle$, and consider the following polynomial

$$
P_{2}(\mu)=(n-1)(\mu+n)\left(\frac{n s-4 \tau+4 n \tau+4}{2} \mu-(n-4)\left(n^{2} \tau+n s-n \tau-s+2\right)\right) .
$$

Since the first term of $P_{2}(\mu)$ is always nonnegative, then we just consider the second term. Let $n=3$, then $P_{2}(\mu)$ is nonnegative if

$$
\left\{\begin{array} { l } 
{ \tau > 2 } \\
{ s > - \frac { 8 } { 3 } \tau - \frac { 4 } { 3 } }
\end{array} \quad \text { or } \quad \left\{\begin{array}{l}
\tau<2 \\
s>-3 \tau-2
\end{array}\right.\right.
$$

And $P_{2}(\mu)$ is non-positive if

$$
\left\{\begin{array} { l } 
{ \tau > 2 } \\
{ s < - 3 \tau - 2 }
\end{array} \quad \text { or } \quad \left\{\begin{array}{l}
\tau<2 \\
s<-\frac{8}{3} \tau-\frac{4}{3}
\end{array}\right.\right.
$$

When $n \geq 4$, do it in the same way as Theorem 1.5. Then we finish the proof of Theorem 1.6. 


\section{References}

[1] Aubin T. Some nonlinear problems in Riemannian geometry. Berlin: Springer-Verlag, 1998

[2] Berger M. and Ebin D. Some decompositions of the space of symmetric tensors on a Riemannian manifold. J. Diff. Geom. 1969, 3: 379-392

[3] Besse A. L. Einstein Manifolds. Berlin: Springer-Verlag, 1987

[4] Blair D. E. Spaces of metrics and curvature functionals, Handbook of differential geometry, North-Holland, Amsterdam, Vol(I): 153-185, 2000

[5] Catino G. Some rigidity results on critical metrics for quadratic functionals. Calc. Var. Partial Differential Equations, 2015, 54(3):2921-2937

[6] Guo X., Li H. and Wei G. On variational formaulas of a conformally invariant functional. Results. Math. 2015, 67: 49-70

[7] Gursky M. and Viaclovsky J. Rigidity and stability of Einstein metrics for quadratic curvature functionals. Journal Für die Reine und Angewandte Mathematik, 2015, 700: 37-91

[8] Kobayashi O. On a conformally invariant functional of the space of Riemannian metrics. J. Math. Soc. Japan, 1984, 37(3): 373-389

[9] Lichnerowicz A. Propagatrurs et commutateurs en relativité gén érale (French). Inst. Hautes Études Sci. Publ. Math. 1961,10: 56 pp

[10] Maity S. On the stability of the $L^{p}$-norm of the Riemannian curvature tensor. Proc. Indian Acad. Sci.(Math. Sci.), 2014, 124(3): 383-409

[11] Muto Y. Curvature and critical Riemannian metric. J. Math. Soc. Japan 1974, 26(4): 686-697

[12] Viaclovsky J. Critical Metrics for Riemannian Curvature Functionals. Geometric analysis, IAS/Park City Math. Ser., 22, Amer. Math. Soc., Providence, RI, 2016, 197-274.

\section{Addresses:}

Weimin Sheng: School of Mathematical Sciences, Zhejiang University, Hangzhou 310027, China. Lisheng Wang: School of Mathematical Sciences, Zhejiang university, Hangzhou 310027, China.

Email: weimins@zju.edu.cn; wlspaper@yeah.net 